\title{
Optimal Strategy Synthesis in Stochastic Müller Games ${ }^{\star, \star \star}$
}

\author{
Krishnendu Chatterjee \\ EECS, University of California, Berkeley, USA \\ c_krish@eecs.berkeley.edu
}

\begin{abstract}
The theory of graph games with $\omega$-regular winning conditions is the foundation for modeling and synthesizing reactive processes. In the case of stochastic reactive processes, the corresponding stochastic graph games have three players, two of them (System and Environment) behaving adversarially, and the third (Uncertainty) behaving probabilistically. We consider two problems for stochastic graph games: the qualitative problem asks for the set of states from which a player can win with probability 1 (almost-sure winning); and the quantitative problem asks for the maximal probability of winning (optimal winning) from each state. We consider $\omega$-regular winning conditions formalized as Müller winning conditions. We present optimal memory bounds for pure almost-sure winning and optimal winning strategies in stochastic graph games with Müller winning conditions. We also present improved memory bounds for randomized almost-sure winning and optimal strategies.
\end{abstract}

\section{Introduction}

A stochastic graph game [6] is played on a directed graph with three kinds of states: player-1, player-2, and probabilistic states. At player-1 states, player 1 chooses a successor state; at player-2 states, player 2 chooses a successor state; and at probabilistic states, a successor state is chosen according to a given probability distribution. The result of playing the game forever is an infinite path through the graph. If there are no probabilistic states, we refer to the game as a 2-player graph game; otherwise, as a 21/2-player graph game. There has been a long history of using 2-player graph games for modeling and synthesizing reactive processes [116: a reactive system and its environment represent the two players, whose states and transitions are specified by the states and edges of a game graph. Consequently, $2^{1 / 2}$-player graph games provide the theoretical foundation for modeling and synthesizing processes that are both reactive and stochastic.

For the modeling and synthesis (or "control") of reactive processes, one traditionally considers $\omega$-regular winning conditions, which naturally express the temporal specifications and fairness assumptions of transition systems [12]. This

\footnotetext{
* This research was supported in part by the the AFOSR MURI grant F49620-00-10327, and the NSF grant CCR-0225610.

$\star \star$ Full proofs available in 2 .
} 
paper focuses on $21 / 2$-player graph games with respect to an important normal form of $\omega$-regular winning conditions; namely Müller winning conditions [17.

In the case of 2-player graph games, where no randomization is involved, a fundamental determinacy result of Gurevich and Harrington 10] based on LAR (latest appearance record) construction ensures that, given an $\omega$-regular winning condition, at each state, either player 1 has a strategy to ensure that the condition holds, or player 2 has a strategy to ensure that the condition does not hold. Thus, the problem of solving 2-player graph games consists in finding the set of winning states, from which player 1 can ensure that the condition holds. Along with the computation of the winning states, the characterization of complexity of winning strategies is a central question, since the winning strategies represent the implementation of the controller in the synthesis problem. The elegant algorithm of Zielonka [18] uses the LAR construction to compute winning sets in 2-player graph games with Müller conditions. In [7] the authors present an insightful analysis of Zielonka's algorithm to present optimal memory bounds (matching upper and lower bound) for winning strategies in 2-player graph games with Müller conditions.

In the case of $21 / 2$-player graph games, where randomization is present in the transition structure, the notion of winning needs to be clarified. Player 1 is said to win surely if she has a strategy that guarantees to achieve the winning condition against all player-2 strategies. While this is the classical notion of winning in the 2-player case, it is less meaningful in the presence of probabilistic states, because it makes all probabilistic choices adversarial (it treats them analogously to player-2 choices). To adequately treat probabilistic choice, we consider the probability with which player 1 can ensure that the winning condition is met. We thus define two solution problems for $21 / 2$-player graph games: the qualitative problem asks for the set of states from which player 1 can ensure winning with probability 1; the quantitative problem asks for the maximal probability with which player 1 can ensure winning from each state (this probability is called the value of the game at a state). Correspondingly, we define almost-sure winning strategies, which enable player 1 to win with probability 1 whenever possible, and optimal strategies, which enable player 1 to win with maximal probability. The main result of this paper is an optimal memory bound for pure (deterministic) almost-sure and optimal strategies in $21 / 2$-player graph games with Müller conditions. In fact we generalize the elegant analysis of [7] to present an upper bound for optimal strategies for $21 / 2$-player graph games with Müller conditions that matches the lower bound for sure winning in 2-player games. As a consequence we generalize several results known for 21/2-player graph games: such as existence of pure memoryless optimal strategies for parity conditions [51914] and Rabin conditions 4 . We present the result for almost-sure strategies in Section 3 and then generalize it to optimal strategies in Section 4 . We also study the memory bounds for randomized strategies. In case of randomized strategies we improve the upper bound for almost-sure and optimal strategies as compared to pure strategies (Section 5). The problem of a matching upper and lower bound for almost-sure and optimal randomized strategies remains open. 


\section{Definitions}

We consider several classes of turn-based games, namely, two-player turn-based probabilistic games (21/2-player games), two-player turn-based deterministic games (2-player games), and Markov decision processes (11/2-player games).

Notation. For a finite set $A$, a probability distribution on $A$ is a function $\delta: A \rightarrow$ $[0,1]$ such that $\sum_{a \in A} \delta(a)=1$. We denote the set of probability distributions on $A$ by $\mathcal{D}(A)$. Given a distribution $\delta \in \mathcal{D}(A)$, we denote by $\operatorname{Supp}(\delta)=\{x \in A \mid$ $\delta(x)>0\}$ the support of $\delta$.

Game graphs. A turn-based probabilistic game graph (21/2-player game graph) $G=\left((S, E),\left(S_{1}, S_{2}, S_{\bigcirc}\right), \delta\right)$ consists of a directed graph $(S, E)$, a partition $\left(S_{1}\right.$, $S_{2}, S_{\bigcirc}$ ) of the finite set $S$ of states, and a probabilistic transition function $\delta$ : $S_{\bigcirc} \rightarrow \mathcal{D}(S)$, where $\mathcal{D}(S)$ denotes the set of probability distributions over the state space $S$. The states in $S_{1}$ are the player-1 states, where player 1 decides the successor state; the states in $S_{2}$ are the player-2 states, where player 2 decides the successor state; and the states in $S_{\bigcirc}$ are the probabilistic states, where the successor state is chosen according to the probabilistic transition function $\delta$. We assume that for $s \in S_{\bigcirc}$ and $t \in S$, we have $(s, t) \in E$ iff $\delta(s)(t)>0$, and we often write $\delta(s, t)$ for $\delta(s)(t)$. For technical convenience we assume that every state in the graph $(S, E)$ has at least one outgoing edge. For a state $s \in S$, we write $E(s)$ to denote the set $\{t \in S \mid(s, t) \in E\}$ of possible successors.

A set $U \subseteq S$ of states is called $\delta$-closed if for every probabilistic state $u \in U \cap S_{\bigcirc}$, if $(u, t) \in E$, then $t \in U$. The set $U$ is called $\delta$-live if for every nonprobabilistic state $s \in U \cap\left(S_{1} \cup S_{2}\right)$, there is a state $t \in U$ such that $(s, t) \in E$. A $\delta$-closed and $\delta$-live subset $U$ of $S$ induces a subgame graph of $G$, indicated by $G \uparrow U$.

The turn-based deterministic game graphs (2-player game graphs) are the special case of the $21 / 2$-player game graphs with $S_{\bigcirc}=\emptyset$. The Markov decision processes $(11 / 2$-player game graphs) are the special case of the $21 / 2$-player game graphs with $S_{1}=\emptyset$ or $S_{2}=\emptyset$. We refer to the MDPs with $S_{2}=\emptyset$ as player-1 $M D P s$, and to the MDPs with $S_{1}=\emptyset$ as player-2 MDPs.

Plays and strategies. An infinite path, or play, of the game graph $G$ is an infinite sequence $\omega=\left\langle s_{0}, s_{1}, s_{2}, \ldots\right\rangle$ of states such that $\left(s_{k}, s_{k+1}\right) \in E$ for all $k \in \mathbb{N}$. We write $\Omega$ for the set of all plays, and for a state $s \in S$, we write $\Omega_{s} \subseteq \Omega$ for the set of plays that start from the state $s$.

A strategy for player 1 is a function $\sigma: S^{*} \cdot S_{1} \rightarrow \mathcal{D}(S)$ that assigns a probability distribution to all finite sequences $\boldsymbol{w} \in S^{*} \cdot S_{1}$ of states ending in a player-1 state (the sequence represents a prefix of a play). Player 1 follows the strategy $\sigma$ if in each player-1 move, given that the current history of the game is $\boldsymbol{w} \in S^{*} \cdot S_{1}$, she chooses the next state according to the probability distribution $\sigma(\boldsymbol{w})$. A strategy must prescribe only available moves, i.e., for all $\boldsymbol{w} \in S^{*}$, and $s \in S_{1}$ we have $\operatorname{Supp}(\sigma(\boldsymbol{w} \cdot s)) \subseteq E(s)$. The strategies for player 2 are defined analogously. We denote by $\Sigma$ and $\Pi$ the set of all strategies for player 1 and player 2, respectively.

Once a starting state $s \in S$ and strategies $\sigma \in \Sigma$ and $\pi \in \Pi$ for the two players are fixed, the outcome of the game is a random walk $\omega_{s}^{\sigma, \pi}$ for which 
the probabilities of events are uniquely defined, where an event $\mathcal{A} \subseteq \Omega$ is a measurable set of paths. Given strategies $\sigma$ for player 1 and $\pi$ for player 2, a play $\omega=\left\langle s_{0}, s_{1}, s_{2}, \ldots\right\rangle$ is feasible if for every $k \in \mathbb{N}$ the following three conditions hold: (1) if $s_{k} \in S_{\bigcirc}$, then $\left(s_{k}, s_{k+1}\right) \in E$; (2) if $s_{k} \in S_{1}$, then $\sigma\left(s_{0}, s_{1}, \ldots, s_{k}\right)\left(s_{k+1}\right)>0$; and $(3)$ if $s_{k} \in S_{2}$ then $\pi\left(s_{0}, s_{1}, \ldots, s_{k}\right)\left(s_{k+1}\right)>0$. Given two strategies $\sigma \in \Sigma$ and $\pi \in \Pi$, and a state $s \in S$, we denote by Outcome $(s, \sigma, \pi) \subseteq \Omega_{s}$ the set of feasible plays that start from $s$ given strategies $\sigma$ and $\pi$. For a state $s \in S$ and an event $\mathcal{A} \subseteq \Omega$, we write $\operatorname{Pr}_{s}^{\sigma, \pi}(\mathcal{A})$ for the probability that a path belongs to $\mathcal{A}$ if the game starts from the state $s$ and the players follow the strategies $\sigma$ and $\pi$, respectively. In the context of player- 1 MDPs we often omit the argument $\pi$, because $\Pi$ is a singleton set.

We classify strategies according to their use of randomization and memory. The strategies that do not use randomization are called pure. A player- 1 strategy $\sigma$ is pure if for all $\boldsymbol{w} \in S^{*}$ and $s \in S_{1}$, there is a state $t \in S$ such that $\sigma(\boldsymbol{w} \cdot s)(t)=1$. We denote by $\Sigma^{P} \subseteq \Sigma$ the set of pure strategies for player 1 . A strategy that is not necessarily pure is called randomized. Let $\mathrm{M}$ be a set called memory, that is, $\mathrm{M}$ is a set of memory elements. A player-1 strategy $\sigma$ can be described as a pair of functions $\sigma=\left(\sigma_{u}, \sigma_{m}\right)$ : a memory-update function $\sigma_{u}$ : $S \times \mathrm{M} \rightarrow \mathrm{M}$ and a next-move function $\sigma_{m}: S_{1} \times \mathrm{M} \rightarrow \mathcal{D}(S)$. We can think of strategies with memory as input/output automaton computing the strategies (see [7] for details). The strategy $\left(\sigma_{u}, \sigma_{m}\right)$ is finite-memory if the memory $\mathrm{M}$ is finite, and then we denote the size of the memory of the strategy $\sigma$ by the size of its memory M, i.e., $|\mathrm{M}|$. We denote by $\Sigma^{F}$ the set of finite-memory strategies for player 1 , and by $\Sigma^{P F}$ the set of pure finite-memory strategies; that is, $\Sigma^{P F}=\Sigma^{P} \cap \Sigma^{F}$. The strategy $\left(\sigma_{u}, \sigma_{m}\right)$ is memoryless if $|\mathrm{M}|=1$; that is, the next move does not depend on the history of the play but only on the current state. A memoryless player-1 strategy can be represented as a function $\sigma: S_{1} \rightarrow \mathcal{D}(S)$. A pure memoryless strategy is a pure strategy that is memoryless. A pure memoryless strategy for player 1 can be represented as a function $\sigma: S_{1} \rightarrow S$. We denote by $\Sigma^{M}$ the set of memoryless strategies for player 1 , and by $\Sigma^{P M}$ the set of pure memoryless strategies; that is, $\Sigma^{P M}=\Sigma^{P} \cap \Sigma^{M}$. Analogously we define the corresponding strategy families $\Pi^{P}, \Pi^{F}, \Pi^{P F}, \Pi^{M}$, and $\Pi^{P M}$ for player 2 .

Given a finite-memory strategy $\sigma \in \Sigma^{F}$, let $G_{\sigma}$ be the game graph obtained from $G$ under the constraint that player 1 follows the strategy $\sigma$. The corresponding definition $G_{\pi}$ for a player-2 strategy $\pi \in \Pi^{F}$ is analogous, and we write $G_{\sigma, \pi}$ for the game graph obtained from $G$ if both players follow the finitememory strategies $\sigma$ and $\pi$, respectively. Observe that given a $21 / 2$-player game graph $G$ and a finite-memory player- 1 strategy $\sigma$, the result $G_{\sigma}$ is a player- 2 MDP. Similarly, for a player-1 MDP $G$ and a finite-memory player-1 strategy $\sigma$, the result $G_{\sigma}$ is a Markov chain. Hence, if $G$ is a $21 / 2$-player game graph and the two players follow finite-memory strategies $\sigma$ and $\pi$, the result $G_{\sigma, \pi}$ is a Markov chain. These observations will be useful in the analysis of $2^{1 / 2}$-player games.

Objectives. An objective for a player consists of an $\omega$-regular set of winning plays $\Phi \subseteq \Omega$ [17]. In this paper we study zero-sum games, where the objectives of the two players are complementary; that is, if the objective of one player 
is $\Phi$, then the objective of the other player is $\bar{\Phi}=\Omega \backslash \Phi$. We consider $\omega$-regular objectives specified as Müller objectives. For a play $\omega=\left\langle s_{0}, s_{1}, s_{2}, \ldots\right\rangle$, let $\operatorname{Inf}(\omega)$ be the set $\left\{s \in S \mid s=s_{k}\right.$ for infinitely many $\left.k \geq 0\right\}$ of states that appear infinitely often in $\omega$. We use colors to define objectives as in 7. A $21 / 2$-player game $(G, C, \chi, \mathcal{F} \subseteq \mathcal{P}(C))$ consists of a $2^{1 / 2}$-player game graph $G$, a finite set $C$ of colors, a partial function $\chi: S \rightarrow C$ that assigns colors to some states, and a winning condition specified by a subset $\mathcal{F}$ of the power set $\mathcal{P}(C)$ of colors. The winning condition defines subset $\Phi \subseteq \Omega$ of winning plays, defined as follows: $\operatorname{Müller}(\mathcal{F})=\{\omega \in \Omega \mid \chi(\operatorname{Inf}(\omega)) \in \mathcal{F}\}$, that is the set of paths $\omega$ such that the colors appearing infinitely often in $\omega$ is in $\mathcal{F}$.

Remarks. A winning condition $\mathcal{F} \subseteq \mathcal{P}(C)$ has a split if there are sets $C_{1}, C_{2} \in \mathcal{F}$ such that $C_{1} \cup C_{2} \notin \mathcal{F}$. A winning condition is a Rabin winning condition if it do not have splits, and it is a Streett winning condition if $\mathcal{P}(C) \backslash \mathcal{F}$ does not have a split. This notions coincide with the Rabin and Streett winning conditions usually defined in the literature (see [15/7] for details). We now define the reachability, safety, Büchi and coBüchi objectives that will be useful in proofs.

- Reachability and safety objectives. Given a set $T \subseteq S$ of "target" states, the reachability objective requires that some state of $T$ be visited. The set of winning plays is thus $\operatorname{Reach}(T)=\left\{\omega=\left\langle s_{0}, s_{1}, s_{2}, \ldots\right\rangle \in \Omega \mid s_{k} \in\right.$ $T$ for some $k \geq 0\}$. Given a set $F \subseteq S$, the safety objective requires that only states of $F$ be visited. Thus, the set of winning plays is $\operatorname{Safe}(F)=\{\omega=$ $\left\langle s_{0}, s_{1}, s_{2}, \ldots\right\rangle \in \Omega \mid s_{k} \in F$ for all $\left.k \geq 0\right\}$.

- Büchi and coBüchi objectives. Given a set $B \subseteq S$ of "Büchi" states, the Büchi objective requires that $B$ is visited infinitely often. Formally, the set of winning plays is $\operatorname{Büchi}(B)=\{\omega \in \Omega \mid \operatorname{Inf}(\omega) \cap B \neq \emptyset\}$. Given $C \subseteq S$, the coBüchi objective requires that all states visited infinitely often are in $C$. Formally, the set of winning plays is $\operatorname{coBüchi}(C)=\{\omega \in \Omega \mid \operatorname{Inf}(\omega) \subseteq C\}$.

Sure, almost-sure, positive winning and optimality. Given a player-1 objective $\Phi$, a strategy $\sigma \in \Sigma$ is sure winning for player 1 from a state $s \in S$ if for every strategy $\pi \in \Pi$ for player 2 , we have Outcome $(s, \sigma, \pi) \subseteq \Phi$. A strategy $\sigma$ is almost-sure winning for player 1 from the state $s$ for the objective $\Phi$ if for every player-2 strategy $\pi$, we have $\operatorname{Pr}_{s}^{\sigma, \pi}(\Phi)=1$. A strategy $\sigma$ is positive winning for player 1 from the state $s$ for the objective $\Phi$ if for every player-2 strategy $\pi$, we have $\operatorname{Pr}_{s}^{\sigma, \pi}(\Phi)>0$. The sure, almost-sure and positive winning strategies for player 2 are defined analogously. Given an objective $\Phi$, the sure winning set $\langle\langle 1\rangle\rangle_{\text {sure }}(\Phi)$ for player 1 is the set of states from which player 1 has a sure winning strategy. Similarly, the almost-sure winning set $\langle\langle 1\rangle\rangle_{\text {almost }}(\Phi)$ and the positive winning set $\langle\langle 1\rangle\rangle_{\text {pos }}(\Phi)$ for player 1 is the set of states from which player 1 has an almost-sure winning and a positive winning strategy, respectively. The sure winning set $\langle\langle 2\rangle\rangle_{\text {sure }}(\Omega \backslash \Phi)$, the almost-sure winning set $\langle\langle 2\rangle\rangle_{\text {almost }}(\Omega \backslash \Phi)$ and the positive winning set $\langle\langle 2\rangle\rangle_{\text {pos }}(\Omega \backslash \Phi)$ for player 2 are defined analogously. It follows from the definitions that for all $21 / 2$-player game graphs and all objectives $\Phi$, we have $\left\langle\langle 1\rangle_{\text {sure }}(\Phi) \subseteq\left\langle\langle 1\rangle_{\text {almost }}(\Phi) \subseteq\langle\langle 1\rangle\rangle_{\text {pos }}(\Phi)\right.\right.$. Computing sure, almost-sure and positive winning sets and strategies is referred to as the qualitative analysis of $21 / 2$-player games 8 . 
Given $\omega$-regular objectives $\Phi \subseteq \Omega$ for player 1 and $\Omega \backslash \Phi$ for player 2 , we define the value functions $\langle\langle 1\rangle\rangle_{v a l}$ and $\langle\langle 2\rangle\rangle_{v a l}$ for the players 1 and 2 , respectively, as the following functions from the state space $S$ to the interval $[0,1]$ of reals: for all states $s \in S$, let $\langle\langle 1\rangle\rangle_{\text {val }}(\Phi)(s)=\sup _{\sigma \in \Sigma} \inf _{\pi \in \Pi} \operatorname{Pr}_{s}^{\sigma, \pi}(\Phi)$ and $\langle\langle 2\rangle\rangle_{\text {val }}(\Omega \backslash \Phi)(s)=$ $\sup _{\pi \in \Pi} \inf _{\sigma \in \Sigma} \operatorname{Pr}_{s}^{\sigma, \pi}(\Omega \backslash \Phi)$. In other words, the value $\langle\langle 1\rangle\rangle_{v a l}(\Phi)(s)$ gives the maximal probability with which player 1 can achieve her objective $\Phi$ from state $s$, and analogously for player 2. The strategies that achieve the value are called optimal: a strategy $\sigma$ for player 1 is optimal from the state $s$ for the objective $\Phi$ if $\langle\langle 1\rangle\rangle_{v a l}(\Phi)(s)=\inf _{\pi \in \Pi} \operatorname{Pr}_{s}^{\sigma, \pi}(\Phi)$. The optimal strategies for player 2 are defined analogously. Computing values and optimal strategies is referred to as the quantitative analysis of $2 \frac{1}{2}$-player games. The set of states with value 1 is called the limit-sure winning set [8]. For $21 / 2$-player game graphs with $\omega$-regular objectives the almost-sure and limit-sure winning sets coincide [4].

Let $\mathcal{C} \in\{P, M, F, P M, P F\}$ and consider the family $\Sigma^{\mathcal{C}} \subseteq \Sigma$ of special strategies for player 1 . We say that the family $\Sigma^{\mathcal{C}}$ suffices with respect to a player-1 objective $\Phi$ on a class $\mathcal{G}$ of game graphs for sure winning if for every game graph $G \in \mathcal{G}$ and state $s \in\langle\langle 1\rangle\rangle_{\text {sure }}(\Phi)$, there is a player-1 strategy $\sigma \in \Sigma^{\mathcal{C}}$ such that for every player-2 strategy $\pi \in \Pi$, we have Outcome $(s, \sigma, \pi) \subseteq \Phi$. Similarly, the family $\Sigma^{\mathcal{C}}$ suffices with respect to the objective $\Phi$ on the class $\mathcal{G}$ of game graphs for (a) almost-sure winning if for every game graph $G \in \mathcal{G}$ and state $s \in\langle\langle 1\rangle\rangle_{\text {almost }}(\Phi)$, there is a player-1 strategy $\sigma \in \Sigma^{\mathcal{C}}$ such that for every player2 strategy $\pi \in \Pi$, we have $\operatorname{Pr}_{s}^{\sigma, \pi}(\Phi)=1$; (b) positive winning if for every game graph $G \in \mathcal{G}$ and state $s \in\langle\langle 1\rangle\rangle_{\text {pos }}(\Phi)$, there is a player-1 strategy $\sigma \in \Sigma^{\mathcal{C}}$ such that for every player-2 strategy $\pi \in \Pi$, we have $\operatorname{Pr}_{s}^{\sigma, \pi}(\Phi)>0$; and (c) optimality if for every game graph $G \in \mathcal{G}$ and state $s \in S$, there is a player-1 strategy $\sigma \in \Sigma^{\mathcal{C}}$ such that $\langle\langle 1\rangle\rangle_{\text {val }}(\Phi)(s)=\inf _{\pi \in \Pi} \operatorname{Pr}_{s}^{\sigma, \pi}(\Phi)$. The notion of sufficiency for size of finite-memory strategies is obtained by referring to the size of the memory $\mathrm{M}$ of the strategies. The notions of sufficiency of strategies for player 2 is defined analogously.

Determinacy. For sure winning, the $1 \frac{1}{2}$-player and $21 / 2$-player games coincide with 2-player (deterministic) games where the random player (who chooses the successor at the probabilistic states) is interpreted as an adversary, i.e., as player 2. Theorem 1 and Theorem 2 state the classical determinacy results for 2 -player and $2^{1 / 2}$-player game graphs with Müller objectives. It follows from Theorem 2 that for all Müller objectives $\Phi$, for all $\varepsilon>0$, there exists an $\varepsilon$ optimal strategy $\sigma_{\varepsilon}$ for player 1 such that for all $\pi$ and all $s \in S$ we have $\operatorname{Pr}_{s}^{\sigma, \pi}(\Phi) \geq\langle\langle 1\rangle\rangle_{v a l}(\Phi)(s)-\varepsilon$.

Theorem 1 (Qualitative determinacy [10]). For all 2-player game graphs and Müller objectives $\Phi$, we have $\langle\langle 1\rangle\rangle_{\text {sure }}(\Phi) \cap\langle\langle 2\rangle\rangle_{\text {sure }}(\Omega \backslash \Phi)=\emptyset$ and $\langle\langle 1\rangle\rangle_{\text {sure }}(\Phi) \cup\langle\langle 2\rangle\rangle_{\text {sure }}(\Omega \backslash \Phi)=S$. Moreover, on 2-player game graphs, the family of pure finite-memory strategies suffices for sure winning with respect to Müller objectives.

Theorem 2 (Quantitative determinacy [13]). For all 21/2-player game graphs, for all Müller winning conditions $\mathcal{F} \subseteq \mathcal{P}(C)$, and all states $s$, we have $\langle\langle 1\rangle\rangle_{\text {val }}(\operatorname{Müller}(\mathcal{F}))(s)+\langle\langle 2\rangle\rangle_{\text {val }}(\Omega \backslash \operatorname{Müller}(\mathcal{F}))(s)=1$. 


\section{Memory Bound for Qualitative Winning Strategies}

In this section we present optimal memory bounds for pure strategies with respect to qualitative (almost-sure and positive) winning for $2^{1 / 2}$-player game graphs with Müller winning conditions. The result is obtained by a generalization of the result of [7] and depends on the novel constructions of Zielonka [18] for 2-player games. In [7 the authors use an insightful analysis of Zielonka's construction to present an upper bound and a matching lower bound on memory of sure winning strategies in 2-player games with Müller objectives. In this section we generalize the result of [7] to show that the same upper bound holds for qualitative winning strategies in $21 / 2$-player games with Müller objectives. We now introduce some notations and the Zielonka tree of a Müller condition.

Notation. Let $\mathcal{F} \subseteq \mathcal{P}(C)$ be a winning condition. For $D \subseteq C$ we define $(\mathcal{F}$ $D) \subseteq \mathcal{P}(D)$ as the set $\left\{D^{\prime} \in \mathcal{F} \mid D^{\prime} \subseteq D\right\}$. For a Müller condition $\mathcal{F} \subseteq \mathcal{P}(C)$ we denote by $\overline{\mathcal{F}}$ the complementary condition, i.e., $\overline{\mathcal{F}}=\mathcal{P}(C) \backslash \mathcal{F}$. Similarly for an objective $\Phi$ we denote by $\bar{\Phi}$ the complementary objective, i.e., $\bar{\Phi}=\Omega \backslash \Phi$.

Definition 1 (Zielonka tree of a winning condition [18]). The Zielonka tree of a winning condition $\mathcal{F} \subseteq \mathcal{P}(C)$, denoted $\mathcal{Z}_{\mathcal{F}, C}$, is defined inductively as follows: (a) if $C \notin \mathcal{F}$, then $\mathcal{Z}_{\mathcal{F}, C}=\mathcal{Z}_{\overline{\mathcal{F}}, C}$, where $\overline{\mathcal{F}}=\mathcal{P}(C) \backslash \mathcal{F}$; and (b) if $C \in \mathcal{F}$, then the root of $\mathcal{Z}_{\mathcal{F}, C}$ is labeled with $C$. Let $C_{0}, C_{1}, \ldots, C_{k-1}$ be all the maximal sets in $\{X \notin \mathcal{F} \mid X \subseteq C\}$. Then we attach to the root, as its subtrees, the Zielonka trees of $\mathcal{F} \uparrow C_{i}$, i.e., $\mathcal{Z}_{\mathcal{F} \backslash C_{i}, C_{i}}$, for $i=0,1, \ldots, k-1$. Hence the Zielonka tree is a tree with nodes labeled by sets of colors. A node of $\mathcal{Z}_{\mathcal{F}, C}$ is a 0 -level node if it is labeled with a set from $\mathcal{F}$, otherwise it is a 1-level node. In the sequel we write $\mathcal{Z}_{\mathcal{F}}$ to denote $\mathcal{Z}_{\mathcal{F}, C}$ if $C$ is clear from the context.

Definition 2 (The number $m_{\mathcal{F}}$ of Zielonka tree). Let $\mathcal{F} \subseteq \mathcal{P}(C)$ be a winning condition and $\mathcal{Z}_{\mathcal{F}_{0}, C_{0}}, \mathcal{Z}_{\mathcal{F}_{1}, C_{1}}, \ldots, \mathcal{Z}_{\mathcal{F}_{k-1}, C_{k-1}}$ be the subtrees attached to the root of the tree $\mathcal{Z}_{\mathcal{F}, C}$, where $\mathcal{F}_{i}=\mathcal{F} \uparrow C_{i} \subseteq \mathcal{P}\left(C_{i}\right)$ for $i=0,1, \ldots, k-1$. We define the number $m_{\mathcal{F}}$ inductively as follows

$$
m_{\mathcal{F}}= \begin{cases}1 & \text { if } \mathcal{Z}_{\mathcal{F}, C} \text { does not have any subtrees } \\ \max \left\{m_{\mathcal{F}_{0}}, m_{\mathcal{F}_{1}}, \ldots, m_{\mathcal{F}_{k-1}}\right\} & \text { if } C \notin \mathcal{F},(1 \text {-level node }) \\ \sum_{i=1}^{k-1} m_{\mathcal{F}_{i}} & \text { if } C \in \mathcal{F},(0 \text {-level node }) .\end{cases}
$$

Our goal is to show that for winning conditions $\mathcal{F}$ pure finite-memory qualitative winning strategies of size $m_{\mathcal{F}}$ exist in $21 / 2$-player games. This proves the upper bound. The results of [7] already established the matching lower bound for 2-player games. This establishes the optimal bound of memory of qualitative winning strategies for $21 / 2$-player games. We start with the key notion of attractors that will be crucial in our proofs.

Definition 3 (Attractors). Given a $2^{1 / 2}$-player game graph $G$ and a set $U \subseteq S$ of states, such that $G \uparrow U$ is a subgame, and $T \subseteq S$ we define $\operatorname{Attr}_{1, \bigcirc}(T, U)$ as follows: $T_{0}=T \cap U$ and for $j \geq 0$ we define $T_{j+1}$ from $T_{j}$ as follows

$$
T_{j+1}=T_{j} \cup\left\{s \in\left(S_{1} \cup S_{\bigcirc}\right) \cap U \mid E(s) \cap T_{j} \neq \emptyset\right\} \cup\left\{s \in S_{2} \cap U \mid E(s) \cap U \subseteq T_{j}\right\} .
$$


and $A=\operatorname{Attr}_{1, \bigcirc}(T, U)=\bigcup_{j \geq 0} T_{j}$. We obtain $\operatorname{Attr}_{2, \bigcirc}(T, U)$ by exchanging the roles of player 1 and player 2. A pure memoryless attractor strategy $\sigma^{A}:(A \backslash$ $T) \cap S_{1} \rightarrow S$ for player 1 on $A$ to $T$ is as follows: for $i>0$ and a state $s \in\left(T_{i} \backslash T_{i-1}\right) \cap S_{1}$, the strategy $\sigma^{A}(s) \in T_{i-1}$ chooses a successor in $T_{i-1}$ (which exists by definition).

Lemma 1 (Attractor properties). Let $G$ be a $2 \frac{1}{2}$-player game graph and $U \subseteq S$ be a set of states such that $G \uparrow U$ is a subgame. For a set $T \subseteq S$ of states, let $Z=\operatorname{Attr}_{1, \bigcirc}(T, U)$. Then the following assertions hold.

1. $G \uparrow(U \backslash Z)$ is a subgame.

2. Let $\sigma^{Z}$ be a pure memoryless attractor strategy for player 1. For all strategies $\pi$ for player 2 in the subgame $G \uparrow U$ and for all states $s \in U$ we have

(a) if $\operatorname{Pr}_{s}^{\sigma^{Z}, \pi}(\operatorname{Reach}(Z))>0$, then $\operatorname{Pr}_{s}^{\sigma^{Z}, \pi}(\operatorname{Reach}(T))>0$; and

(b) if $\operatorname{Pr}_{s}^{\sigma^{Z}, \pi}(\operatorname{Büchi}(Z))>0$, then $\operatorname{Pr}_{s}^{\sigma^{Z}, \pi}(\operatorname{Büchi}(T) \mid \operatorname{Büchi}(Z))=1$.

We now present the main result of this section (upper bound on memory for qualitative winning strategies).

Theorem 3 (Qualitative forgetful determinacy). Let $(G, C, \chi, \mathcal{F})$ be a

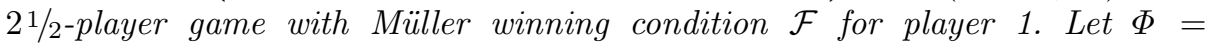
$\operatorname{Müller}(\mathcal{F})$, and consider the following sets

$W_{1}^{>0}=\langle\langle 1\rangle\rangle_{\text {pos }}(\Phi) ; \quad W_{1}=\langle\langle 1\rangle\rangle_{\text {almost }}(\Phi) ; \quad W_{2}^{>0}=\langle\langle 2\rangle\rangle_{\text {pos }}(\bar{\Phi}) ; \quad W_{2}=\langle\langle 2\rangle\rangle_{\text {almost }}(\bar{\Phi})$.

The following assertions hold.

1. We have (a) $W_{1}^{>0} \cup W_{2}=S$ and $W_{1}^{>0} \cap W_{2}=\emptyset$; and (b) $W_{2}^{>0} \cup W_{1}=S$ and $W_{2}^{>0} \cap W_{1}=\emptyset$.

2. (a) Player 1 has a pure strategy $\sigma$ with memory of size $m_{\mathcal{F}}$ such that for all states $s \in W_{1}^{>0}$ and for all strategies $\pi$ for player 2 we have $\operatorname{Pr}_{s}^{\sigma, \pi}(\Phi)>0$; and (b) player 2 has a pure strategy $\pi$ with memory of size $m_{\overline{\mathcal{F}}}$ such that for all states $s \in W_{2}$ and for all strategies $\sigma$ for player 1 we have $\operatorname{Pr}_{s}^{\sigma, \pi}(\bar{\Phi})=1$.

3. (a) Player 1 has a pure strategy $\sigma$ with memory of size $m_{\mathcal{F}}$ such that for all states $s \in W_{1}$ and for all strategies $\pi$ for player 2 we have $\operatorname{Pr}_{s}^{\sigma, \pi}(\Phi)=1$; and (b) player 2 has a pure strategy $\pi$ with memory of size $m_{\overline{\mathcal{F}}}$ such that for all states $s \in W_{2}^{>0}$ and for all strategies $\sigma$ for player 1 we have $\operatorname{Pr}_{s}^{\sigma, \pi}(\bar{\Phi})>0$.

Proof. The first part of the result is a consequence of Theorem 2. We will concentrate on the proof for the result for part 2. The last part (part 3) follows from a symmetric argument.

The proof goes by induction on the structure of the Zielonka tree $\mathcal{Z}_{\mathcal{F}, C}$ of the winning condition $\mathcal{F}$. We assume that $C \notin \mathcal{F}$. The case when $C \in \mathcal{F}$ can be proved by a similar argument: if $C \in \mathcal{F}$, then we consider $\widehat{c} \notin C$ and consider the winning condition $\widehat{\mathcal{F}}=\mathcal{F} \subseteq \mathcal{P}(C \cup\{\widehat{c}\})$ with $C \cup\{\widehat{c}\} \notin \widehat{\mathcal{F}}$. Hence we consider, without loss of generality, that $C \notin \mathcal{F}$ and let $C_{0}, C_{1}, \ldots, C_{k-1}$ be the label of the subtrees attached to the root $C$, i.e., $C_{0}, C_{1}, \ldots, C_{k-1}$ are maximal subset of colors that appear in $\mathcal{F}$. We will define by induction a non-decreasing sequence of sets $\left(U_{j}\right)_{j \geq 0}$ as follows. Let $U_{0}=\emptyset$ and for $j>0$ we define $U_{j}$ below: 
1. $A_{j}=\operatorname{Attr}_{1, O}\left(U_{j-1}, S\right)$ and $X_{j}=S \backslash A_{j}$;

2. $D_{j}=C \backslash C_{j} \bmod k$ and $Y_{j}=X_{j} \backslash \operatorname{Attr}_{2, \bigcirc}\left(\chi^{-1}\left(D_{j}\right), X_{j}\right)$;

3. let $Z_{j}$ be the set of positive winning states for player 1 in $(G$ $Y_{j}, C_{j \bmod k}, \chi, \mathcal{F}\left\lceil C_{j} \bmod k\right)$, (i.e., $Z_{j}=\left\langle\langle 1\rangle_{\text {pos }}(\operatorname{Müller}(\mathcal{F}\rceil\right.$ $\left.\left.C_{j} \bmod k\right)\right)$ in $\left.G \backslash Y_{j}\right)$; hence $\left(Y_{j} \backslash Z_{j}\right)$ is almost-sure winning for player 2 in the subgame; and

4. $U_{j}=A_{j} \cup Z_{j}$.

We start with an observation.

Observation 1. For all $s \in S_{2} \cap Z_{j}$, we have $E(s) \subseteq Z_{j} \cup A_{j}$. This follows from the following case analysis.

- Since $Y_{j}$ is a complement of an attractor set $\operatorname{Attr}_{2, \bigcirc} \bigcirc\left(\chi^{-1}\left(D_{j}\right), X_{j}\right)$, it follows that for all states $s \in S_{2} \cap Y_{j}$ we have $E(s) \cap X_{j} \subseteq Y_{j}$. It follows that $E(s) \subseteq Y_{j} \cup A_{j}$.

- Since player 2 can win almost-surely from the set $Y_{j} \backslash Z_{j}$, if a state $s \in Y_{j} \cap S_{2}$ has an edge to $Y_{j} \backslash Z_{j}$, then $s \in Y_{j} \backslash Z_{j}$. Hence for $s \in S_{2} \cap Z_{j}$ we have $E(s) \cap\left(Y_{j} \backslash Z_{j}\right)=\emptyset$.

We will denote by $\mathcal{F}_{i}$ the winning condition $\mathcal{F}\left\lceil C_{i}\right.$, for $i=0,1, \ldots, k-1$, and $\overline{\mathcal{F}}_{i}=\mathcal{P}\left(C_{i}\right) \backslash \mathcal{F}_{i}$. By induction hypothesis on $\mathcal{F}_{i}=\mathcal{F}\left\lceil C_{j} \bmod k\right.$, player 1 has a pure positive winning strategy of size $m_{\mathcal{F}_{i}}$ from $Z_{j}$ and player 2 has a pure almost-sure winning strategy of size $m_{\overline{\mathcal{F}}_{i}}$ from $Y_{j} \backslash Z_{j}$. Let $W=\bigcup_{j \geq 0} U_{j}$. We will show in Lemma 2 that player 1 has a pure positive winning strategy of size $m_{\mathcal{F}}$ from $W$; and then in Lemma 3 we will show that player 2 has a pure almost-sure winning strategy of size $m_{\overline{\mathcal{F}}}$ from $S \backslash W$. This completes the proof. We now prove the Lemmas 2 and 3 .

Lemma 2. Player 1 has a pure positive winning strategy of size $m_{\mathcal{F}}$ from $W$.

Proof. By induction hypothesis on $U_{j-1}$ player 1 has a pure positive winning strategy $\sigma_{j-1}^{U}$ of size $m_{\mathcal{F}}$ from $U_{j-1}$. From the set $A_{j}=\operatorname{Attr}_{1, \bigcirc}\left(U_{j-1}, S\right)$, player 1 has a pure memoryless attractor strategy $\sigma_{j}^{A}$ to bring the game to $U_{j-1}$ with positive probability (Lemma 1(part 2.(a))), and then use $\sigma_{j-1}^{U}$ and ensure winning with positive probability from the set $A_{j}$. Let $\sigma_{j}^{Z}$ be the pure positive winning strategy for player 1 in $Z_{j}$ of size $m_{\mathcal{F}_{i}}$, where $i=j \bmod k$. We now show the combination of strategies $\sigma_{j-1}^{U}, \sigma_{j}^{A}$ and $\sigma_{j}^{Z}$ ensure positive probability winning for player 1 from $U_{j}$. If the play starts at a state $s \in Z_{j}$, then player 1 follows $\sigma_{j}^{Z}$. If the play stays in $Y_{j}$ for ever, then the strategy $\sigma_{j}^{Z}$ ensures that player 1 wins with positive probability. By observation 1 of Theorem 3 , for all states $s \in Y_{j} \cap S_{2}$, we have $E(s) \subseteq Y_{j} \cup A_{j}$. Hence if the play leaves $Y_{j}$, then player 2 must chose an edge to $A_{j}$. In $A_{j}$ player 1 can use the attractor strategy $\sigma_{j}^{A}$ followed by $\sigma_{j-1}^{U}$ to ensure positive probability win. Hence if the play is in $Y_{j}$ for ever with probability 1 , then $\sigma_{j}^{Z}$ ensures positive probability win, and if the play reaches $A_{j}$ with positive probability, then $\sigma_{j}^{A}$ followed by $\sigma_{j-1}^{U}$ ensures positive probability win.

We now formally present $\sigma_{j}^{U}$ defined on $U_{j}$. Let $\sigma_{j}^{Z}=\left(\sigma_{j, u}^{Z}, \sigma_{j, m}^{Z}\right)$ be the strategy obtained from inductive hypothesis; defined on $Z_{j}$ (i.e., arbitrary elsewhere) 
of size $m_{\mathcal{F}_{i}}$, where $i=j \bmod k$, and ensure winning with positive probability on $Z_{j}$. Let $\sigma_{j, u}^{Z}$ be the memory-update function and $\sigma_{j, m}^{Z}$ be the next-move function of $\sigma_{j}^{Z}$. We assume the memory $M_{\mathcal{F}_{i}}$ of $\sigma_{j}^{Z}$ to be the set $\left\{1,2, \ldots, m_{\mathcal{F}_{i}}\right\}$. The strategy $\sigma_{j}^{A}:\left(A_{j} \backslash U_{j-1}\right) \cap S_{1} \rightarrow A_{j}$ is a pure memoryless attractor strategy on $A_{j}$ to $U_{j-1}$. The strategy $\sigma_{j}^{U}$ is as follows: the memory-update function and the next-move function is

$$
\begin{gathered}
\sigma_{j, u}^{U}(s, m)= \begin{cases}\sigma_{j-1, u}^{U}(s, m) & s \in U_{j-1} \\
\sigma_{j-1, u}^{Z}(s, m) & s \in Z_{j}, m \in M_{\mathcal{F}_{i}} \\
1 & \text { otherwise. }\end{cases} \\
\sigma_{j, m}^{U}(s, m)= \begin{cases}\sigma_{j-1, m}^{U}(s, m) & s \in U_{j-1} \cap S_{1} \\
\sigma_{j-1, m}^{Z}(s, m) & s \in Z_{j} \cap S_{1}, m \in M_{\mathcal{F}_{i}} \\
\sigma_{j-1, m}^{Z}(s, 1) & s \in Z_{j} \cap S_{1}, m \notin M_{\mathcal{F}_{i}} \\
\sigma_{j}^{A}(s) & s \in\left(A_{j} \backslash U_{j-1}\right) \cap S_{1} .\end{cases}
\end{gathered}
$$

The strategy $\sigma_{j}^{U}$ formally defines the strategy described and proves the result.

Lemma 3. Player 2 has a pure almost-sure winning strategy of size $m_{\overline{\mathcal{F}}}$ from $S \backslash W$.

Proof. Let $\ell \in \mathbb{N}$ be such that $\ell \bmod k=0$ and $W=U_{\ell-1}=U_{\ell}=U_{\ell+1}=$ $\cdots=U_{\ell+k-1}$. From the equality $W=U_{\ell-1}=U_{\ell}$ we have $\operatorname{Attr}_{1, \bigcirc}(W, S)=W$. Let us denote by $\bar{W}=S \backslash W$. Hence $G \backslash \bar{W}$ is a subgame (by Lemma 1), and also for all $s \in \bar{W} \cap\left(S_{1} \cup S_{\bigcirc}\right)$ we have $E(s) \subseteq \bar{W}$. The equality $U_{\ell+i-1}=U_{\ell+i}$ implies that $Z_{\ell+i}=\emptyset$. Hence for all $i=0,1, \ldots, k-1$, we have $Z_{\ell+i}=\emptyset$. By inductive hypothesis for all $i=0,1, \ldots, k-1$, player 2 has a pure almost-sure winning strategy $\pi^{i}$ of size $m_{\overline{\mathcal{F}}_{i}}$ in the game $\left(G \nmid Y_{\ell+i}, C_{i}, \chi, \mathcal{F}\left\lceil C_{i}\right)\right.$.

We now describe the construction of a pure almost-sure winning strategy $\pi^{*}$ for player 2 in $\bar{W}$. For $D_{i}=C \backslash C_{i}$ we denote by $\widehat{D}_{i}=\chi^{-1}\left(D_{i}\right)$ the set of states with colors $D_{i}$. If the play starts in a state in $Y_{\ell+i}$, for $i=0,1, \ldots, k-1$, then player 2 uses the almost-sure winning strategy $\pi^{i}$. If the play leaves $Y_{\ell+i}$, then the play must reach $\bar{W} \backslash Y_{\ell+i}=\operatorname{Attr}_{2, \bigcirc}\left(\widehat{D}_{i}, \bar{W}\right)$, since player 1 and random states do not have edges to $W$. In $\operatorname{Attr}_{2,} \bigcirc\left(\widehat{D}_{i}, \bar{W}\right)$, player 2 plays a pure memoryless attractor strategy to reach the set $\widehat{D}_{i}$ with positive probability. If the set $\widehat{D}_{i}$ is reached, then a state in $Y_{(\ell+i+1)} \bmod k$ or in $\operatorname{Attr}_{2, \bigcirc}\left(\widehat{D}_{(i+1) \bmod k}, \bar{W}\right)$ is reached. If $Y_{(\ell+i+1)} \bmod k$ is reached $\pi^{(i+1)} \bmod k$ is followed, and otherwise the pure memoryless attractor strategy to reach the set $\widehat{D}_{(i+1)} \bmod k$ with positive probability is followed. Of course, the play may leave $Y_{(\ell+i+1)} \bmod k$, and reach $Y_{(\ell+i+2)} \bmod k$, and then we would repeat the reasoning, and so on. Let us analyze various cases to prove that $\pi^{*}$ is almost-sure winning for player 2 .

1. If the play finally settles in some $Y_{\ell+i}$, for $i=0,1, \ldots, k-1$, then from this moment player 2 follows $\pi^{i}$ and ensures that the objective $\bar{\Phi}$ is satisfied with probability 1 . Formally, for all states $s \in \bar{W}$, for all strategies $\sigma$ for player 1 
we have $\operatorname{Pr}_{s}^{\sigma, \pi^{*}}\left(\bar{\Phi} \mid \operatorname{coBüchi}\left(Y_{\ell+i}\right)\right)=1$. This holds for all $i=0,1, \ldots, k-1$ and hence for all states $s \in \bar{W}$, for all strategies $\sigma$ for player 1 we have $\operatorname{Pr}_{s}^{\sigma, \pi^{*}}\left(\bar{\Phi} \mid \bigcup_{0 \leq i \leq k-1} \operatorname{coBüchi}\left(Y_{\ell+i}\right)\right)=1$.

2. Otherwise, for all $i=0,1, \ldots, k-1$, the set $\bar{W} \backslash Y_{\ell+i}=\operatorname{Attr}_{2, \bigcirc}\left(\widehat{D}_{i}, \bar{W}\right)$ is visited infinitely often. By Lemma 1, given $\operatorname{Attr}_{2, \bigcirc}\left(\widehat{D}_{i}, \bar{W}\right)$ is visited infinitely often, then the attractor strategy ensures that the set $\widehat{D}_{i}$ is visited infinitely often with probability 1 . Formally, for all states $s \in \bar{W}$, for all strategies $\sigma$ for player 1 , for all $i=0,1, \ldots, k-1$, we have $\operatorname{Pr}_{s}^{\sigma, \pi^{*}}\left(\operatorname{Büchi}\left(\widehat{D}_{i}\right) \mid \operatorname{Büchi}(\bar{W} \backslash\right.$ $\left.\left.Y_{\ell+i}\right)\right)=1$; and also $\operatorname{Pr}_{s}^{\sigma, \pi^{*}}\left(\operatorname{Büchi}\left(\widehat{D}_{i}\right) \mid \bigcap_{0 \leq i \leq k-1} \operatorname{Büchi}\left(\bar{W} \backslash Y_{\ell+i}\right)\right)=1$. It follows that for all states $s \in \bar{W}$, for all strategies $\sigma$ for player 1 we have $\operatorname{Pr}_{s}^{\sigma, \pi^{*}}\left(\bigcap_{0 \leq i \leq k-1} \operatorname{Büchi}\left(\widehat{D}_{i}\right) \mid \bigcap_{0 \leq i \leq k-1} \operatorname{Büchi}\left(\bar{W} \backslash Y_{\ell+i}\right)\right)=1$. Hence the play visits states with colors not in $C_{i}$ with probability 1 . Hence the set of colors visited infinitely often is not contained in any $C_{i}$. Since $C_{0}, C_{1}, \ldots, C_{k-1}$ are all the maximal subsets of $\mathcal{F}$, we have the set of colors visited infinitely often is not in $\mathcal{F}$ with probability 1 , and hence player 2 wins almost-surely.

Hence it follows that for all strategies $\sigma$ and for all states $s \in(S \backslash W)$ we have $\operatorname{Pr}_{s}^{\sigma, \pi^{*}}(\bar{\Phi})=1$. To complete the proof we present precise description of the strategy $\pi^{*}$ with memory of size $m_{\overline{\mathcal{F}}}$. Let $\pi^{i}=\left(\pi_{u}^{i}, \pi_{m}^{i}\right)$ be an almost-sure winning strategy for player 2 for the subgame on $Y_{\ell+i}$ with memory $M_{\overline{\mathcal{F}}_{i}}$. By definition we have $m_{\overline{\mathcal{F}}}=\sum_{i=0}^{k-1} m_{\overline{\mathcal{F}}_{i}}$. Let $M_{\overline{\mathcal{F}}}=\bigcup_{i=0}^{k-1}\left(M_{\overline{\mathcal{F}}_{i}} \times\{i\}\right)$. This set is not exactly the set $\left\{1,2, \ldots, m_{\mathcal{F}}\right\}$, but has the same cardinality (which suffices for our purpose). We define the strategy $\pi^{*}$ as follows:

$$
\begin{aligned}
& \pi_{u}^{*}(s,(m, i))= \begin{cases}\pi_{u}^{i}(s,(m, i)) & s \in Y_{\ell+i} \\
(1, i+1 \bmod k) & \text { otherwise. }\end{cases} \\
& \pi_{m}^{*}(s,(m, i))= \begin{cases}\pi_{m}^{i}(s,(m, i)) & s \in Y_{\ell+i} \\
\pi^{L_{i}}(s) & s \in L_{i} \backslash \widehat{D}_{i} \\
s_{i} & s \in \widehat{D}_{i}, s_{i} \in E(s) \cap \bar{W} .\end{cases}
\end{aligned}
$$

where $L_{i}=\operatorname{Attr}_{2, \bigcirc}\left(\widehat{D}_{i}, \bar{W}\right) ; \pi^{L_{i}}$ is a pure memoryless attractor strategy on $L_{i}$ to $\widehat{D}_{i}$, and $s_{i}$ is a successor state of $s$ in $\bar{W}$ (such a state exists since $\bar{W}$ induces a subgame). This formally represents $\pi^{*}$ and the size of $\pi^{*}$ satisfies the required bound. Observe that the disjoint sum of all $M_{\overline{\mathcal{F}}_{i}}$ was required since $Y_{\ell}, Y_{\ell+1}, \ldots, Y_{\ell+k-1}$ may not be disjoint and the strategy $\pi^{*}$ need to know which $Y_{j}$ the play is in.

Lower bound. In [7] the authors show a matching lower bound for sure winning strategies in 2-player games. In 2-player games any pure almost-sure winning or any pure positive winning strategy is also a sure winning strategy. This observation along with the result of [7] gives us the following result.

Theorem 4 (Lower bound [7]). For all Müller winning conditions $\mathcal{F} \subseteq$ $\mathcal{P}(C)$, there is a 2-player game $(G, C, \chi, \mathcal{F})$ (with a 2-player game graph $G$ ) such 
that every pure almost-sure and positive winning strategy for player 1 requires memory of size at least $m_{\mathcal{F}}$; and every pure almost-sure and positive winning strategy for player 2 requires memory of size at least $m_{\overline{\mathcal{F}}}$.

\section{Memory Bound for Optimal Strategies}

In this section we extend the sufficiency results for families of strategies from almost-sure winning to optimality with respect to all Müller objectives. In the following, we fix a $2^{1 /} / 2$-player game graph $G$. We first present some definitions.

Definition 4 (Value classes). Given a Müller objective $\Phi$, for every real $r \in[0,1]$ the value class with value $r$ is $\operatorname{VC}(\Phi, r)=\left\{s \in S \mid\langle\langle 1\rangle\rangle_{\text {val }}(\Phi)(s)=r\right\}$ is the set of states with value $r$ for player 1 . For $r \in[0,1]$ we denote by $\operatorname{VC}(\Phi,>r)=\bigcup_{q>r} \operatorname{VC}(\Phi, q)$ the value classes greater than $r$ and by $\operatorname{VC}(\Phi,<$ $r)=\bigcup_{q<r} \operatorname{VC}(\Phi, q)$ the value classes smaller than $r$.

Definition 5 (Boundary probabilistic states). Given a value class $\mathrm{VC}(\Phi, r)$, a state $s \in \mathrm{VC}(\Phi, r) \cap S_{\bigcirc}$ is a boundary probabilistic state if $E(s) \cap(S \backslash \operatorname{VC}(\Phi, r)) \neq \emptyset$, i.e., the probabilistic state has an edge out of the value class. For a value class $\operatorname{VC}(\Phi, r)$ we denote by $\operatorname{Bnd}(\Phi, r)$ the set of boundary probabilistic states of value class $r$.

Observation. For a state $s \in \operatorname{Bnd}(\Phi, r)$ we have $E(s) \cap \operatorname{VC}(\Phi,>r) \neq \emptyset$ and $E(s) \cap \operatorname{VC}(\Phi,<r) \neq \emptyset$, i.e., the boundary probabilistic states have edges to higher and lower value classes. It follows that for all Müller objectives $\Phi$ we have $\operatorname{Bnd}(\Phi, 1)=\emptyset$ and $\operatorname{Bnd}(\Phi, 0)=\emptyset$.

Reduction of a value class. Given a value class $\operatorname{VC}(\Phi, r)$, let $\operatorname{Bnd}(\Phi, r)$ be the set of boundary probabilistic states in $\operatorname{VC}(\Phi, r)$. We denote by $G_{\mathrm{Bnd}(\Phi, r)}$ the subgame where every boundary probabilistic state in $\operatorname{Bnd}(\Phi, r)$ is converted to an absorbing state (state with a self-loop). We denote by $G_{\Phi, r}=G_{\operatorname{Bnd}(\Phi, r)}$ $\mathrm{VC}(\Phi, r)$ : this is a subgame since every value class is $\delta$-live, and $\delta$-closed as all states in $\operatorname{Bnd}(\Phi, r)$ are converted to absorbing states.

Lemma 4 (Almost-sure reduction). Let $G$ be a $2^{1 / 2-p l a y e r ~ g a m e ~ g r a p h ~ a n d ~}$ $\mathcal{F} \subseteq \mathcal{P}(C)$ be a Müller winning condition. Let $\Phi=\operatorname{Müller}(\mathcal{F})$. For $0<r<1$, the following assertions hold.

1. Player 1 wins almost-surely for objective $\Phi \cup \operatorname{Reach}(\operatorname{Bnd}(\Phi, r))$ from all states in $G_{\Phi, r}$, i.e., $\langle\langle 1\rangle\rangle_{\text {almost }}(\Phi \cup \operatorname{Reach}(\operatorname{Bnd}(\Phi, r)))=\mathrm{VC}(\Phi, r)$ in $G_{\Phi, r}$.

2. Player 2 wins almost-surely for objective $\bar{\Phi} \cup$ Reach $(\operatorname{Bnd}(\Phi, r))$ from all states in $G_{\Phi, r}$, i.e., $\langle\langle 2\rangle\rangle_{\text {almost }}(\bar{\Phi} \cup \operatorname{Reach}(\operatorname{Bnd}(\Phi, r)))=\operatorname{VC}(\Phi, r)$ in $G_{\Phi, r}$.

Lemma 5 (Almost-sure to optimality [4]). Let $G$ be a $21 / 2$-player game graph and $\mathcal{F} \subseteq \mathcal{P}(C)$ be a Müller winning condition. Let $\Phi=\operatorname{Müller}(\mathcal{F})$. Let $\sigma$ be a strategy such that $(a) \sigma$ is an almost-sure winning strategy from the almostsure winning states $\left(\langle\langle 1\rangle\rangle_{\text {almost }}(\Phi)\right.$ in $\left.G\right)$; and $(b) \sigma$ is an almost-sure winning strategy for objective $\Phi \cup \operatorname{Reach}(\operatorname{Bnd}(\Phi, r))$ in the game $G_{\Phi, r}$, for all $0<r<1$. Then $\sigma$ is an optimal strategy. 
Müller reduction for $G_{\Phi, r}$. Given a Müller winning condition $\mathcal{F}$ and the objective $\Phi=\operatorname{Müller}(\mathcal{F})$, we consider the game $G_{\Phi, r}$ with the objective $\Phi \cup$ $\operatorname{Reach}(\operatorname{Bnd}(\Phi, r))$ for player 1 . We present a simple reduction to a game with objective $\Phi$. The reduction is achieved as follows: without loss of generality we assume $\mathcal{F} \neq \emptyset$, and let $F \in \mathcal{F}$ and $F=\left\{c_{1}^{F}, c_{2}^{F}, \ldots, c_{f}^{F}\right\}$. We construct a game graph $\widetilde{G}_{\Phi, r}$ with objective $\Phi$ for player 1 as follows: convert every state $s_{j} \in \operatorname{Bnd}(\Phi, r)$ to a cycle $U_{j}=\left\{s_{1}^{j}, s_{2}^{j}, \ldots, s_{f}^{j}\right\}$ with $\chi\left(s_{i}^{j}\right)=c_{i}^{F}$, i.e., once $s_{j}$ is reached the cycle $U_{j}$ is repeated with $\chi\left(U_{j}\right) \in \mathcal{F}$. An almost-sure winning strategy in $G_{\Phi, r}$ with objective $\Phi \cup \operatorname{Reach}(\operatorname{Bnd}(\Phi, r))$, is an almost-sure winning strategy in $\widetilde{G}_{\Phi, r}$ with objective $\Phi$; and vice-versa. The present reduction along with Lemma 4 and Lemma 5 gives us Lemma 6. Lemma 6]along with Theorem 3 gives us Theorem 5 .

Lemma 6. For all Müller winning conditions $\mathcal{F}$, the following assertions hold.

1. If the family of pure finite-memory strategies of size $\ell_{\mathcal{F}}^{P}$ suffices for almostsure winning on 21/2-player game graphs, then the family of pure finitememory strategies of size $\ell_{\mathcal{F}}^{P}$ suffices for optimality on $21 / 2$-player game graphs.

2. If the family of randomized finite-memory strategies of size $\ell_{\mathcal{F}}^{R}$ suffices for almost-sure winning on 21/2-player game graphs, then the family of randomized finite-memory strategies of size $\ell_{\mathcal{F}}^{R}$ suffices for optimality on $21 / 2$-player game graphs.

Theorem 5. For all Müller winning conditions $\mathcal{F}$, the family of pure finitememory strategies of size $m_{\mathcal{F}}$ suffices for optimality on $2^{1 / 2-p l a y e r ~ g a m e ~ g r a p h s . ~}$

\section{$5 \quad$ An Improved Bound for Randomized Strategies}

We now show that if a player plays randomized strategies, then the upper bound on memory for optimal strategies can be improved. We first present the notions of an upward closed restriction of a Zielonka tree. The number $m_{\mathcal{F}}^{U}$ of such restrictions of the Zielonka tree will be in general lower than the number $m_{\mathcal{F}}$ of Zielonka trees, and we show that randomized strategies with memory of size $m_{\mathcal{F}}^{U}$ suffices for optimality.

Upward closed sets. A set $\mathcal{F} \subseteq \mathcal{P}(C)$ is upward closed if for all $F \in \mathcal{F}$ and all $F \subseteq F_{1}$ we have $F_{1} \in \mathcal{F}$, i.e., if a set $F$ is in $\mathcal{F}$, then all supersets $F_{1}$ of $F$ are in $\mathcal{F}$ as well.

Upward closed restriction of Zielonka tree. The upward closed restriction of a Zielonka tree for a Müller winning condition $\mathcal{F} \subseteq \mathcal{P}(C)$, denoted as $\mathcal{Z}_{\mathcal{F}, C}^{U}$, is obtained by making upward closed conditions as leaves. Formally, we define $\mathcal{Z}_{\mathcal{F}, C}^{U}$ inductively as follows:

1. if $\mathcal{F}$ is upward closed, then $\mathcal{Z}_{\mathcal{F}, C}^{U}$ is leaf labeled $\mathcal{F}$ (i.e., it has no subtrees);

2. otherwise 
(a) if $C \notin \mathcal{F}$, then $\mathcal{Z}_{\mathcal{F}, C}^{U}=\mathcal{Z}_{\overline{\mathcal{F}}, C}^{U}$, where $\overline{\mathcal{F}}=\mathcal{P}(C) \backslash \mathcal{F}$.

(b) if $C \in \mathcal{F}$, then the root of $\mathcal{Z}_{\mathcal{F}, C}^{U}$ is labeled with $C$; and let $C_{0}, C_{1}, \ldots, C_{k-1}$ be all the maximal sets in $\{X \notin \mathcal{F} \mid X \subseteq C\}$; then we attach to the root, as its subtrees, the Zielonka upward closed restricted trees $\mathcal{Z}_{\mathcal{F}, C}^{U}$ of $\mathcal{F}\left\lceil C_{i}\right.$, i.e., $\mathcal{Z}_{\mathcal{F} \mid C_{i}, C_{i}}^{U}$, for $i=0,1, \ldots, k-1$.

The number $m_{\mathcal{F}}^{U}$ for $\mathcal{Z}_{\mathcal{F}, C}^{U}$ is the number defined as the number $m_{\mathcal{F}}$ was defined for the tree $\mathcal{Z}_{\mathcal{F}, C}$.

We will prove randomized strategies of size $m_{\mathcal{F}}^{U}$ suffices for optimality. To prove this result, we first prove that randomized strategies of size $m_{\mathcal{F}}^{U}$ suffices for almost-sure winning. The result then follows from Lemma 6. To prove the result for almost-sure winning we take a closer look at the proof of Theorem 3 The inductive proof characterizes that if existence of randomized memoryless strategies can be proved for 21/2-player games with Müller winning conditions that appear in the leaves of the Zielonka tree, then the inductive proof generalizes to give a bound as in Theorem 3 . Hence to prove an upper bound of size $m_{\mathcal{F}}^{U}$ for almost-sure winning, it suffices to show that randomized memoryless strategies suffices for upward closed Müller winning conditions. In [3] it was shown that for all $21 / 2$-player games randomized memoryless strategies suffices for almost-sure winning for upward closed objectives. This gives us Theorem 6

Theorem 6. For all Müller winning conditions $\mathcal{F}$, the family of randomized finite-memory strategies of size $m_{\mathcal{F}}^{U}$ suffices for optimality on $2^{1 / 2}$-player game graphs.

Remark. In general we have $m_{\mathcal{F}}^{U}<m_{\mathcal{F}}$. Consider for example $\mathcal{F} \subseteq \mathcal{P}(C)$, where $C=\left\{c_{1}, c_{2}, \ldots, c_{k}\right\}$. For the Müller winning condition $\mathcal{F}=\{C\}$. We have $m_{\mathcal{F}}^{U}=1$, and $m_{\mathcal{F}}=|C|$.

\section{Conclusion}

In this work we present optimal memory bounds for pure almost-sure, positive and optimal strategies for $21 / 2$-player games with Müller winning conditions. We also present improved memory bounds for randomized strategies. Unlike the results of [7] our results do not extend to infinite state games: for example, the results of $[9$ showed that even for $21 / 2$-player pushdown games optimal strategies need not exist, and for $\varepsilon>0$ even $\varepsilon$-optimal strategies may require infinite memory. For lower bound of randomized strategies the constructions of [7] do not work: in fact for the family of games used for lower bounds in 7 randomized memoryless almost-sure winning strategies exist. However, it is known that there exist Müller winning conditions $\mathcal{F} \subseteq \mathcal{P}(C)$, such that randomized almost-sure winning strategies may require memory $|C|$ ! [11. However, whether a matching lower bound of size $m_{\mathcal{F}}^{U}$ can be proved in general, or whether the upper bound of $m_{\mathcal{F}}^{U}$ can be improved and a matching lower bound can be proved for randomized strategies with memory remains open. 


\section{References}

1. J.R. Büchi and L.H. Landweber. Solving sequential conditions by finite-state strategies. Transactions of the AMS, 138:295-311, 1969.

2. K. Chatterjee The complexity of stochastic Müller games. Technical report: UC Berkeley, EECS-2006-141.

3. K. Chatterjee, L. de Alfaro, and T.A. Henzinger. Trading memory for randomness. In QEST'04 IEEE Computer Society Press, 2004.

4. K. Chatterjee, L. de Alfaro, and T.A. Henzinger. The complexity of stochastic Rabin and Streett games. In ICALP'05 vol. 3580 of $L N C S$, pages 878-890. Springer.

5. K. Chatterjee, M. Jurdziński, and T.A. Henzinger. Quantitative stochastic parity games. In SODA'04, pages 114-123. SIAM, 2004.

6. A. Condon. The complexity of stochastic games. Information and Computation, 96:203-224, 1992.

7. S. Dziembowski, M. Jurdziński, and I. Walukiewicz. How much memory is needed to win infinite games? In LICS'97, pages 99-110. IEEE, 1997.

8. L. de Alfaro and T.A. Henzinger. Concurrent $\omega$-regular games. In $L I C S$ '00, pages 141-154. IEEE, 2000.

9. K. Etessami and M. Yannakakis Recursive Markov decision processes and recursive stochastic games. In ICALP'05 vol. 3580 of $L N C S$, pages 891-903. Springer.

10. Y. Gurevich and L. Harrington. Trees, automata, and games. In STOC'82, pages 60-65. ACM, 1982.

11. R. Majumdar. Symbolic algorithms for verification and control. PhD Thesis, UC Berkeley, 2003.

12. Z. Manna and A. Pnueli. The Temporal Logic of Reactive and Concurrent Systems: Specification. Springer, 1992.

13. D.A. Martin. The determinacy of Blackwell games. Journal of Symbolic Logic, 63:1565-1581, 1998.

14. A.K. McIver and C.C. Morgan. Games, probability, and the quantitative $\mu$-calculus $q m \mu$. In LPAR'02, volume 2514 of $L N A I$, pages 292-310. Springer, 2002.

15. D. Niwiński Fixed-point characterization of infinite behavior of finite-state systems. In Theoretical Computer Science, 189(1-2): 1-69, 1997.

16. P.J. Ramadge and W.M. Wonham. Supervisory control of a class of discrete-event processes. SIAM Journal of Control and Optimization, 25:206-230, 1987.

17. W. Thomas. Languages, automata, and logic. In Handbook of Formal Languages, volume 3 (Beyond Words), pages 389-455. Springer, 1997.

18. W. Zielonka Infinite games on finitely coloured graphs with applications to automata on infinite trees. In Theoretical Computer Science, 200(1-2): 135-183, 1998.

19. W. Zielonka. Perfect-information stochastic parity games. In FoSSaCS'04, volume 2987 of LNCS, pages 499-513. Springer, 2004. 